\title{
Morse 势函数与鉄单晶临界切应力的温度依賴关系*
}

\author{
龙期威 邹本三姜 健 \\ （中国科学院金属研究所）
}

在以往的鉬单晶工作报告 ${ }^{[1]}$ 中，我们利用杂质 原子和位错中心原子成键的概念，使用 Morse 势 推得的重要结论之一是: 体心立方金属临界切应力 的溫度依赖关系应该表现为 Morse 势特点的复 杂 函数关棌; 在合适的溫度范 围 内（约 在 $100^{\circ} \mathrm{K}$ $500^{\circ} \mathrm{K}$ 之间）则近似为指数规律。关于后者，已经 指出，和我们鉬单晶实验结果符合。复杂函数和指 数关系在极低溫度附近 $\left(<100^{\circ} \mathrm{K}\right)$ 偏离较大。因此 把理论分析结果和包括有极低溫度附近监界切应力 的实验数据相比较，对于原子间力模型有重要意 义。以往的单晶数据，或者溫度做的不够低，或者 实验点太少, 数据分散, 很难进行比较。本文利用 Morse 势函数进行数值计算, 扭与 Edmondson ${ }^{[2]}$ 和 Cox 等 ${ }^{[3]}$ 的鉄单晶实验数据相比较。结果指出，鉄 单晶临界切应力的溫店依赖关系很好地符合原子键 力模型所导出的规律性。

杂质原子和运动位错中心原子成键的形成和破 坏过程，对位错运动起障碍作用，克服这个障碍需 裂一定的应力，这个应力師摩擦应力。初步近似， 可以只考虛位错中心原子和杂质原子的键 合作 用 （考虑杂质原子和其他近邻的键合作用时，因为键 的性质相同，规律性也不会变)。采用 Morse 势函 数代表键合作用:

$$
u_{c}=D\left\{e^{-2 a\left(r-r_{0}\right)}-2 e^{-a\left(r-r_{0}\right)}\right\}
$$

其中 $r_{0}$ 代表平衡状态下杂质原子和位错中心原子 的距离, 受切应力作用后变为 $r_{0}$ 。 $D$ 表示平衡状态 下的 $u_{c}$ 值， $\alpha$ 为 Morse 势圂数参数。根据文献 [1]
可以将 (1)式在适当的近似条件下写成:

$$
u_{c}=D\left\{e^{-2 A x^{2}-2 B T}-2 e^{-A x^{2}-B T}\right\}
$$

其中 $A=\frac{\alpha}{2 r_{0}}, B=\frac{\alpha}{2 r_{0}}\left(\frac{\vec{u}^{2}}{T}\right)$ 。临界切应力为:

$$
\sigma_{c}=s^{-1}\left(\frac{\partial u_{c}}{\partial x}\right)_{\operatorname{six}}
$$

这里 $S=\left[\frac{\left(c_{i} n\right)^{1 / 3}}{b}\right]^{-1}$ 为具有面积量纲的常数。 $c_{i}$ 是杂质原子浓度(原子百分数)， $n$ 是单位体积中原 子个数(包括溶质原子和溶剂原子)， $b$ 为柏格斯矢 量。对于任意给定的温度, $\left(\frac{\partial u_{c}}{\partial x}\right)_{\text {max }}$ 的 $x$ 由 $\frac{\partial^{2} u_{c}}{\partial x^{2}}=0$ 条件决定，郎

$$
B T=-\ln \left[\frac{2 A x_{c}^{2}-1}{4 A x_{c}^{2}-1}\right]-A x_{c}^{2}
$$

由(2)及 $(3)$, 可得:

$$
\sigma_{c}=2\left(\frac{x_{c}}{r_{0}}\right)\left\{e^{-2 A x_{c}^{2}-2 B T}-e^{-. A x_{c}^{2}-B T}\right\} \alpha D S^{-1}
$$

式中 $x_{c}$ 滿足 (4)，参数 $A 、 B 、 \alpha 、 D$ 原则上都可以由 微观的分子量子理论及位错线段的热振动理论 ${ }^{[4]}$ 算 出, 再根据 (5) 与(4) 式就可以得到 $\sigma_{c}$ 与溫度的关 系。可是目前决定这些参数的一些微观㟥量还沒有 准确测定的数值, 量子力学的计算及涉及到很大的 工作量, 求出绝对值和实验比较是不现实的。一般 采取牛经验的方式, 由实验确定一些参数, 比较其 规律性。

（1）利用在不太低溫度范围 $\ln \sigma_{c}(T)=\ln \sigma_{c}(O)$

*本交會于 1964 年 8 月在长春召开的中国科学院全翼 电子论学习讨论会上报告过。 
- $B T$ 的近似公式 [1]，朵用最小二乘法处理这个温 度范围內的实验数据, 定出 $\ln \sigma_{c}-T$ 图上斜率 $B$ 值。对于 Edmondson ${ }^{[2]}$ 和 $\operatorname{Cox}$ 等 $^{[3]}$ 的 $B$ 值分別为 $1.17 \times 10^{-2}\left({ }^{\circ} \mathrm{K}^{-1}\right)$ 和 $8.7 \times 10^{-3}\left({ }^{\circ} \mathrm{K}^{-1}\right) 。$

（2）假设某一实验值符合理论计算结果（如设 Edmondson 的 $223^{\circ} \mathrm{K}$; Cox 等人的 $143^{\circ} \mathrm{K}$ ), 利用(5) 式定出 $\left(\alpha D S^{-1}\right)$ 值分別为83.7(㫓/时 $\left.{ }^{2}\right)$ 及 $85.5(1000$

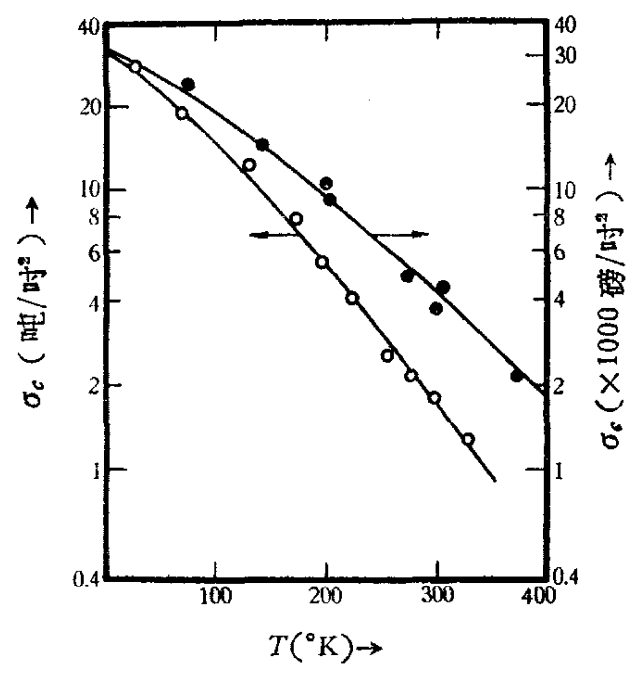

图 1 铁单昆的 $\sigma_{c}-T$ 曲线 (实线为理论曲线)

- Edmondson - Cox et al
磅 $/$ 时 $\left.^{2}\right)$ 。图 1 给出了理论计算曲线和实验点 符 合 的情况。

可以看出，利用杂质原子和位错中心原子成键 的概念推得的临界切应力的温度依赖关系, 理论和 实验数据是符合的。

再由于

$$
\frac{\partial}{\partial T} \ln \sigma_{c}(T)=-\frac{B}{2 A x_{c}^{2}}
$$

对于一般 $B$ 值的情形, 如 $B=10^{-2}\left({ }^{\circ} \mathrm{K}^{-1}\right)$ 时, $153^{\circ} \mathrm{K}$ 的 $A x_{c}^{2}=0.58 ; 412^{\circ} \mathrm{K}$ 的 $A x_{c}^{2}=0.505$, 可见在这 个溫度范围內上式接近于一常数 $-B$, 郎 $\sigma_{c}$ 的溫度 依赖关系近似指数规律。这一点和我们过去鎮单昆 临界切应力的实验规律符合。

[1] 龙期威、何青, 中国物理学会 1963 年年会论交摘 要，42 页; 中国科学，1964 年，第 13 焱，第 1 期, 160 页。

[2] Edmondson B., Proc. Roy. Soc., 264, 176 (1961). 参阅: Allen N. P., Fracture, 130 (1959).

[3] Cox J. J., Horne G. T. and Mehl R. F., Trans. $A S M .$, 49, 116 (1957).

[4] Leibfried G., in: Dislocation and Mechanical Properties of crystals, p. 495 (1957).

\section{来函照登}

编辑同志:

贵刊 1964 年 11 月号第 1003 页 “二烷基亚磷酸氫酯的合成”一文， 应作如下的更正:

表 1 中产率\%项第 4 行应加入 92 ;

右栏反应式应改为

$$
5 \mathrm{ROH}+2 \mathrm{PCl}_{8}+\mathrm{H}_{2} \mathrm{O} \stackrel{15-20^{\circ}}{\longrightarrow} 2(\mathrm{RO})_{2} \mathrm{POH}+5 \mathrm{HCl}+\mathrm{RCl}
$$

蔡继英 陈桂明 